Check for updates

Cite this: J. Mater. Chem. A, 2017, 5 , 22305

Received 7th June 2017

Accepted 2nd October 2017

DOI: $10.1039 / \mathrm{c} 7 \mathrm{ta0} 4959 \mathrm{e}$

rsc.li/materials-a

\section{Confined crystallization of a HKUST-1 metal- organic framework within mesostructured silica with enhanced structural resistance towards water $\dagger$}

\author{
M. Mazaj, (D) *a T. Čendak, ${ }^{a}$ G. Buscarino, ${ }^{b}$ M. Todaro (DD bc and N. Zabukovec Logar ${ }^{\text {ad }}$
}

A HKUST-1 metal-organic framework was crystallized in the $\mathrm{NH}_{2}$-modified mesostructured silica FDU-12 in order to improve its structural stability upon water exposure. In-depth structural characterization studies of the designed composite confirmed successful formation of the MOF phase within the ordered spherical mesopores of the silica matrix. In spite of the confinement within the cavities, MOF exhibits full accessibility for the adsorbed gas molecules. In contrast to the bulk HKUST-1, which undergoes slow phase transition in a humid environment, the structural integrity of the HKUST-1 in the humid-protective matrix remains unchanged even after immersion and stirring in water at elevated temperature.

\section{Introduction}

Metal-organic frameworks (MOFs) are a fast developing class of porous materials constructed from the nodes of metal ions and multidentate organic ligands forming crystalline hybrid nets. The ability to design structures with high external surface areas, void accessibilities and high density of accessible active sites gives MOFs great potential for applications especially in the fields of adsorption, separation and catalysis. ${ }^{1-7}$ Implementation of MOFs for industrial processes such as heterogeneous catalysis, wastewater treatment, heat storage or flue gas purification, is to a large extent dependent on their structural stability in a humid environment. In contrast to some MOF systems (e.g. UiO-66(Zr), MIL-140(Zr), MIL-100(Al), MIL-53(Al), MIL-53(Cr), MIL-100(Cr), MIL-101(Cr), etc.) with extremely high structural resistance towards water, $^{\mathbf{8 - 1 2}}$ there are many MOFs which undergo irreversible structural transformation or complete degradation upon water exposure. Structural stability in a humid environment is generally considered to be driven by the tendency to exchange coordinatively metal-bonded ligands with water molecules. ${ }^{9,13}$ The degree of exchangeability depends on many chemical features such as metal valence, ${ }^{9}$ charge-tosize ratio of metal cations, ${ }^{14,15}$ Brønsted basicity ${ }^{16,17}$ as well as the overall dimensionality and ligand-to-metal connectivity. ${ }^{9}$

${ }^{a}$ National Institute of Chemistry, Hajdrihova 19, 1000 Ljubljana, Slovenia. E-mail: matjaz.mazaj@ki.si

${ }^{b}$ Dipartimento di Fisica e Chimica, Università di Palermo, 90123 Palermo, Italy 'Dipartimento di Fisica e Astronomia, Università di Catania, 95123 Catania, Italy ${ }^{d}$ University of Nova Gorica, Vipavska 13, 5000 Nova Gorica, Slovenia

$\dagger$ Electronic supplementary information (ESI) available: SEM micrographs, MOF content calculations, HR-TEM and SAED images, gas sorption isotherms, water sorption isotherms. See DOI: 10.1039/c7ta04959e
Several strategies have already been used to overcome the issues of' structural instability of MOFs in a humid environment. ${ }^{18}$ One of the most commonly used approaches is ligand functionalization by hydrophobic functional groups which can be achieved either by employing functionalized ligands as a starting synthesis precursor or by post-synthetic chemical modification. ${ }^{19-30}$ Hydrophobicity can be increased with the modification of the crystal surface as well thus preventing water from entering the pores. This can be achieved for instance by coating with amorphous carbon or by adsorption of surfactants. ${ }^{31-33}$ Another interesting approach is cation exchange. Cations with a high tendency to replace coordinately bonded ligands with water molecules can be partially exchanged with less hydrophilic ones in order to enhance the resistance of the MOF framework toward water. ${ }^{34}$

All of the above described strategies of improving hydrostability deal with functionalizing bulk MOF structures which can be sometimes hard to achieve due to the crystal lattice energy barriers or structural sensitivity to chemical modification processes. Alternatively, the physico-chemical properties of MOFs can be tuned or enhanced by their integration with other functional materials. ${ }^{35,36}$ Immobilization of MOFs on a silica nanoporous support is a promising way to improve their chemical resistance towards water and to design hierarchical porous systems with unique MOF-intrinsic structural and chemical properties. Formation of different MOF systems within disordered or ordered (MCM-41, SBA-15) mesoporous silica matrices has already been reported. ${ }^{37-45}$ Confined crystallization of MOFs is however limited to a large extent by the textural properties of silica matrices. To ensure efficient formation of MOFs within nanoporous silica, some parameters have to be taken into account: (a) the dimensions of mesopores 
should provide sufficient space so that formation energy can be compromised with the nanoparticle growth (sufficient space for the crystallization of nanoparticles with energetically stable minimum size); ${ }^{\mathbf{6 , 4 7}}$ (b) for efficient and uniform filling of mesopores with the MOF phase, spherical shapes are preferred over the cylindrical ones since nanoparticles initially tend to grow in a spherical manner; ${ }^{48}$ (c) the three-dimensional pore system of the silica matrix is preferable over the twodimensional one, due to the better diffusivity and accessibility to the MOF sorption sites; (d) MOF crystallization seems to be more controllable within the ordered mesoporous systems than in the disordered ones where the crystal growth on the surface of the silica matrix can be difficult to prevent. ${ }^{37}$ Herein, we report on a specially designed MOF/silica composite, where a HKUST-1 phase $\left(\mathrm{Cu}_{3}(\mathrm{BTC})_{2}\right)$, presenting one of the most frequently investigated humid-sensitive MOFs, crystallized within an ultra-large mesoporous FDU-12 silica matrix with a cubic pore arrangement. This type of silica support was carefully chosen considering the above mentioned criteria for textural parameters. With different in-depth complementary studies, successful confined crystallization of the MOF phase within silica cavities was proven. The composite exhibits significantly improved hydrothermal structural stability and unobstructed sorption accessibility in comparison with the pristine HKUST-1.

\section{Experimental}

\section{Synthesis procedures}

$\mathrm{NH}_{2}$-FDU-12 matrix. An ultra-large pore FDU-12 matrix with an fcc pore arrangement was synthesized according to the published procedure. ${ }^{49}$ Typically, $1 \mathrm{~g}$ of the Pluronic F127 triblock copolymer (Sigma-Aldrich) and $2.5 \mathrm{~g}$ of $\mathrm{KCl}$ (99.9\%, Sigma Aldrich) were dissolved in $60 \mathrm{~mL}$ of $2 \mathrm{M} \mathrm{HCl} \mathrm{(37 \% ,} \mathrm{Sigma-}$ Aldrich). $6 \mathrm{~mL}$ of ethylbenzene (99.9\%, Fluka), acting as a swelling agent, was added to the solution and stirred overnight at $14{ }^{\circ} \mathrm{C}$. Finally, $4.8 \mathrm{~mL}$ of tetraethylorthosilicate $(98 \%$ TEOS, Sigma-Aldrich) was added and the mixture was again stirred overnight at $14{ }^{\circ} \mathrm{C}$ before it was transferred into a Teflonlined stainless-steel autoclave and treated at $180{ }^{\circ} \mathrm{C}$ for 4 hours. The obtained product was filtered, dried at $60{ }^{\circ} \mathrm{C}$ in air and calcined at $550{ }^{\circ} \mathrm{C} .1 \mathrm{~g}$ of the calcined product was hydrothermally treated in $2 \mathrm{M} \mathrm{HCl}$ for 4 days at $130^{\circ} \mathrm{C}$ in order to enlarge the pore volume and calcined again at $550{ }^{\circ} \mathrm{C}$.

Generation of amine binding sites on the surface of the FDU12 silica framework was performed by post-synthesis grafting with (3-aminopropyl)triethoxysilane (99\% APTES, SigmaAldrich). $1 \mathrm{~g}$ of the calcined FDU-12 product was degassed in a Schlenk line at $100{ }^{\circ} \mathrm{C}$ for 2 hours in vacuum prior to the addition of $200 \mathrm{~mL}$ of toluene (99\% Sigma-Aldrich) and $0.6 \mathrm{~mL}$ of APTES and stirred at room temperature overnight. The product is henceforth denoted as $\mathrm{NH}_{2}$-FDU-12. The nitrogen content of $0.9 \mathrm{wt} \%$ determined by $\mathrm{CHN}$ analysis corresponds to the $\mathrm{NH}_{2}$ group density of $0.6 \mathrm{mmol} \mathrm{g}{ }^{-1}$ of silica matrix.

HKUST-1@NH $\mathrm{N}_{2}$-FDU-12. First, the optimum amount of $\mathrm{Cu}^{2+}$ was grafted on $\mathrm{NH}_{2}$-FDU-12 by stirring $1 \mathrm{~g}$ of the matrix in the solution prepared from $3.14 \mathrm{~g}(0.013 \mathrm{~mol})$ of $\mathrm{Cu}\left(\mathrm{NO}_{3}\right)_{2} \cdot 3 \mathrm{H}_{2} \mathrm{O}$
(99\%, Fluka) dissolved in a mixture of $160 \mathrm{~mL}$ of ethanol (99.9\%, Sigma-Aldrich) and $40 \mathrm{~mL}$ of demineralized water for 24 hours. This intermediate material is denoted as $\mathrm{Cu}-\mathrm{NH}_{2}-\mathrm{FDU}-$ $12.1 \mathrm{~g}$ of the grafted product was transferred into a stainlesssteel Teflon-lined autoclave and mixed with the solution of $0.40 \mathrm{~g}$ of 1,3,5-benzenetricarboxylic acid (95\% BTC, SigmaAldrich) in $3.5 \mathrm{~mL}$ of water and $13.5 \mathrm{~mL}$ of ethanol (EtOH). The amount of BTC added was stoichiometrically calculated according to the amount of $\mathrm{Cu}^{2+}$ within the silica matrix, which was found to be $5.5 \mathrm{wt} \%$ according to the elemental analysis. The reaction mixture was solvothermally treated under microwave irradiation at $150{ }^{\circ} \mathrm{C}$ for 20 minutes. The final product denoted as HKUST-1/FDU-12 was recovered by filtration and dried under ambient conditions.

Bulk HKUST-1. In order to perform some comparative studies, bulk HKUST-1 with different crystallite sizes was also prepared with the modified published procedures. ${ }^{50}$ Typically, $0.15 \mathrm{~g}$ of $\mathrm{Cu}\left(\mathrm{NO}_{3}\right)_{2} \cdot 3 \mathrm{H}_{2} \mathrm{O}$ and $0.14 \mathrm{~g}$ of BTC were added to $10 \mathrm{~mL}$ of an EtOH/water mixture with a $4: 1$ volumetric ratio. The reaction mixture was solvothermally treated under microwave irradiation at $130{ }^{\circ} \mathrm{C}$ for 20 minutes. The product was recovered by filtration and dried under ambient conditions.

\section{Characterization methods}

$\mathrm{X}$-ray powder diffraction data of the samples were collected on a PANalytical X'Pert PRO high-resolution diffractometer with $\mathrm{CuK}_{\alpha} 1$ radiation $(\lambda=1.5406 \AA)$ in the range from 5 to $60^{\circ} 2 \theta$ with the step of $0.034^{\circ}$ per $100 \mathrm{~s}$ using a fully opened 100 channel $\mathrm{X}$ 'Celerator detector. The morphological properties of the samples were observed by scanning electron microscopy measurements (SEM) on a Zeiss Supra ${ }^{\mathrm{TM}}$ 3VP field-emission gun (FEG) microscope. Elemental analysis was performed by energy dispersive X-ray analysis (EDAX) with an INCA Energy system attached to the above described microscope and using a Perkin Elmer 2400 Series II CHNS analyzer. The thermal analysis (TG/DTG) was performed on a Q5000 IR thermogravimeter (TA Instruments, Inc.). The measurements were carried out in an air flow $\left(10 \mathrm{~mL} \mathrm{~min}^{-1}\right)$ with the heating rate of $10{ }^{\circ} \mathrm{C} \min ^{-1}$.

All gas sorption measurements were performed on an IMIHTP manometric sorption analyzer (Hiden Isochema, Inc.). The specific surface areas were determined by the BET method based on the $\mathrm{N}_{2}$ sorption isotherms measured at $77 \mathrm{~K}$. Pore size distribution analysis (PSD) was performed using the NLDFT procedure based on the adsorption data. $\mathrm{H}_{2}, \mathrm{CO}_{2}$ and $\mathrm{CH}_{4}$ sorption measurements were performed at specified temperatures and pressures. Prior to sorption analysis, each sample was evacuated at $150{ }^{\circ} \mathrm{C}$ for 16 hours.

High-resolution transmission electron microscopy (HRTEM) was performed on a $200 \mathrm{kV}$ field-emission gun (FEG) microscope JEOL JEM 2100. Samples were dispersed in ethanol and placed on a copper holey carbon grid. The specimens were additionally coated with carbon in order to prevent excessive charging of the samples under the electron beam.

${ }^{13} \mathrm{C}$ spin-echo, ${ }^{1} \mathrm{H}^{-13} \mathrm{C}$ and ${ }^{1} \mathrm{H}^{-29} \mathrm{Si}$ CPMAS NMR spectra were recorded on a $600 \mathrm{MHz}$ Varian NMR System equipped with 
a $1.6 \mathrm{~mm}$ NB Triple Resonance HXY FastMAS probe and a $3.2 \mathrm{~mm}$ Varian NB Double Resonance HX MAS probe. The Larmor frequencies for ${ }^{13} \mathrm{C}$ and ${ }^{29} \mathrm{Si}$ nuclei were 150.812 and 119.131 MHz, respectively. Chemical shifts of both nuclei were reported relative to the signals of these nuclei in tetramethylsilane. ${ }^{1} \mathrm{H}-{ }^{13} \mathrm{C}$ and ${ }^{1} \mathrm{H}-{ }^{29} \mathrm{Si}$ CPMAS measurements were executed at $10 \mathrm{kHz}$ sample rotation frequency with relaxation delay of $3 \mathrm{~s}$ and cross-polarization contact time of $4 \mathrm{~ms} .{ }^{13} \mathrm{C}$ spin-echo measurement was executed at $36 \mathrm{kHz}$ sample rotation frequency with relaxation delay of $50 \mathrm{~ms}$ and echo delay time of $27.78 \mu \mathrm{s}$.

Electron paramagnetic resonance (EPR) measurements were recorded using a Bruker EMX-micro spectrometer in the X-band (about 9.5 GHz) and with a magnetic-field modulation frequency of $100 \mathrm{kHz}$. The spectra were acquired by putting the glass tube containing the sample under study into a Dewar flask. The latter was filled with liquid nitrogen for measurements at $77 \mathrm{~K}$, whereas it was empty (unfilled with liquid nitrogen) for measurements at room temperature $(300 \mathrm{~K})$. The samples were activated under vacuum at $120{ }^{\circ} \mathrm{C}$ for $6 \mathrm{~h}$ before EPR measurements.

\section{Results and discussion}

The prepared composite material was first characterized by diffraction, sorption, microscopic and spectroscopic techniques in order to reliably determine the structural correlation between the MOF phase and the silica matrix. After thorough structural analysis, the accessibility to HKUST-1 adsorption sites located within the mesoporous silica was evaluated by gas sorption analysis. Experiments on the structural resistivity of the MOF towards water were performed in the final stage.

\section{Structural property investigations}

The X-ray powder diffraction pattern of the HKUST-1/FDU-12 composite shows a good match of the reflections with the pure HKUST-1 material indicating the presence of the MOF phase within the composite. Increase of the background in the range between $15^{\circ}$ and $35^{\circ} 2 \theta$ is due to the presence of the amorphous walls of silica (FDU-12 matrix). The broadening of the reflections and decrease of the intensities, which is clearly visible in the case of HKUST-1/FDU-12 when compared to the pattern of pure HKUST-1, is a strong indication of the formation of a nanosized MOF phase. The particle size calculated using the Scherrer equation is found to be $28 \mathrm{~nm}$. Nanosized crystallization is indeed expected in the confined space of $30 \mathrm{~nm}$ in diameter, available in the large-pore FDU-12 matrix. Additionally, SEM observations did not reveal any separate crystals of HKUST-1 within the composite material (Fig. S1 $\dagger$ ).

The HKUST-1 content within the silica matrix was calculated considering the elemental analysis of the composite and the mesopore volume of the FDU-12- $\mathrm{NH}_{2}$ matrix. HKUST-1/FDU-12 contained $5.5 \mathrm{wt} \%$ of $\mathrm{Cu}$ as determined by EDS analysis. Considering the chemical formula of HKUST-1, $\mathrm{Cu}_{3}(\mathrm{BTC})_{2^{-}}$ $\cdot 3 \mathrm{H}_{2} \mathrm{O}$, it is possible to estimate that the composite contains approximately $20 \mathrm{wt} \%$ of HKUST-1. Additionally, taking into account the mesopore volume of the $\mathrm{NH}_{2}$-FDU-12 matrix determined from $\mathrm{N}_{2}$ sorption isotherm analysis using the $\mathrm{BJH}$ method, it can be estimated that approximately half of the total available mesopores are filled with the HKUST-1 phase (details of the occupancy calculations are available in the ESI $\dagger$ ). Attempts to increase the amount of MOF phase within the matrix by using higher concentrations of $\mathrm{Cu}^{2+}$ (up to $0.1 \mathrm{M}$ ), by increasing the $\mathrm{pH}$ value of the $\mathrm{Cu}^{2+}$ solution (from 3 to 5 ) in the grafting process or by addition of higher amounts of the BTC ligand to the $\mathrm{Cu}-\mathrm{NH}_{2}$-FDU-12 sample were unsuccessful since separate crystals of HKUST-1 started to appear along with the silica matrix (Fig. S2 $\dagger$ ). Incorporation of the MOF within the mesopores seems to exert certain degree of stress on the whole mesostructure. Thus, the occupation of one half of the mesopores seems to be an optimal loading which still allows complete crystallization within the cavities. At this point however, MOF occupancy calculations are merely estimations, since EDS analysis cannot provide any reliable information about the structural correlation between the MOF and the pores of the matrix, so further analyses on the textural properties need to be done. Nevertheless, the obtained findings are a starting point for further discussion in the text.

Thermogravimetric analysis (Fig. 1b) was performed to confirm the composition and compare the thermal properties of the composite with the silica matrix and bulk HKUST-1 material. The $\mathrm{NH}_{2}$-FDU-12 matrix shows gradual weight loss over the whole temperature region up to $600{ }^{\circ} \mathrm{C}$ without any notable step. The final loss of $13.5 \mathrm{wt} \%$ could be attributed to the desorption of small quantities of physisorbed water and the degradation of APTES grafted on the silica matrix. Bulk HKUST1 shows weight losses in two steps. First, the gradual step up to $120{ }^{\circ} \mathrm{C}$ is due to the removal of the adsorbed and coordinated
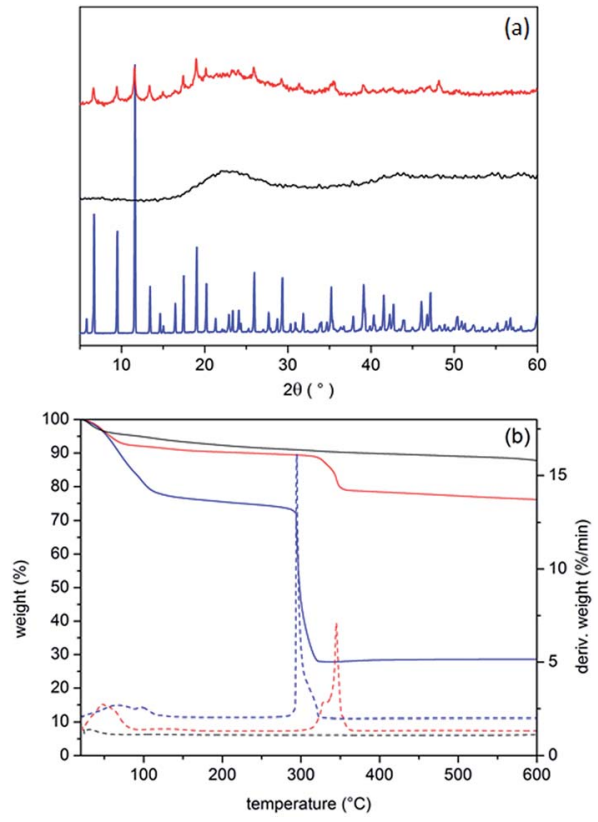

Fig. 1 (a) X-ray powder diffraction patterns and (b) TG/DTG analysis curves. $\mathrm{NH}_{2}$-FDU-12 (black lines), HKUST-1/FDU-12 composite (red lines) and bulk HKUST-1 (blue lines). 
water $(28.6 \mathrm{wt} \%)$ and the second, very distinctive step at $300{ }^{\circ} \mathrm{C}$ is due to the degradation of the framework. Similarly, HKUST-1/ FDU-12 loses weight in two steps. From the weight loss in the second step it can be calculated that the composite contains $21 \mathrm{wt} \%$ of the HKUST-1 phase, which is in good agreement with the EDS-based calculations. It is interesting to observe that the decomposition of the HKUST-1 phase within the composite is shifted by $50{ }^{\circ} \mathrm{C}$ towards higher temperatures with respect to the bulk HKUST-1. The thermal stabilization of the MOF phase could be the consequence of the confined crystallization within the silica mesopores which inhibits framework degradation.

$\mathrm{N}_{2}$ sorption isotherms and pore size distribution (PSD) analyses of $\mathrm{NH}_{2}$-FDU-12, bulk HKUST-1 and the composite material are shown in Fig. 2, whereas the corresponding textural data are given in Table $1 . \mathrm{NH}_{2}$-FDU-12 shows a type IV isotherm with pronounced desorption hysteresis typical of 'inkbottle' shaped mesopores with narrowed entrances and negligible contribution of the micropores. PSD analysis shows bimodal pore size distribution assigned to the spherical cavities with the average diameter of $22 \mathrm{~nm}$ which are linked to each other with narrower window openings with the size of $4 \mathrm{~nm}$. The slightly reduced size of the FDU-12 mesopore cavities when compared to the literature could be assigned to the modification of the silica with APTES. ${ }^{49,51}$ On the other hand, bulk
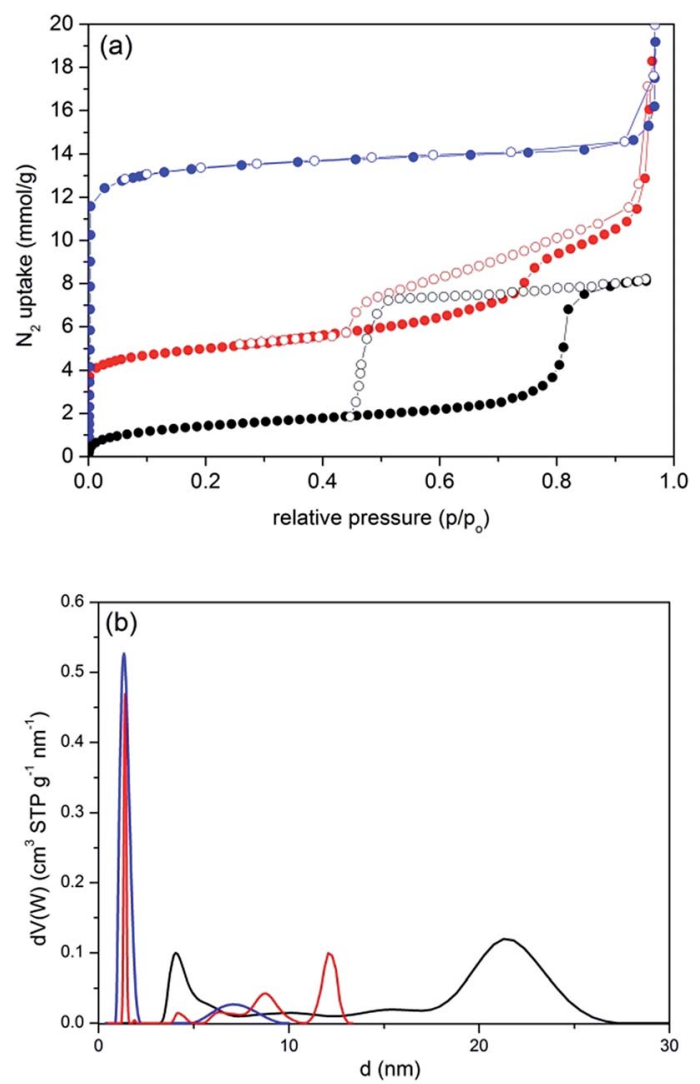

Fig. 2 (a) $\mathrm{N}_{2}$ sorption isotherms and (b) pore size distribution analyses of $\mathrm{NH}_{2}$-FDU-12 (black), HKUST-1/FDU-12 composite (red) and bulk HKUST-1 (blue) recorded at $77 \mathrm{~K}$. Full squares - adsorption points, empty squares - desorption points.
HKUST-1 exhibits a typical type I isotherm with narrow pore size distribution of the micropores. The isotherm of the composite material shows a similar shape to the isotherm of the $\mathrm{NH}_{2}$-FDU12 silica matrix, but with significant increase of the micropore contribution and BET surface area (from 72 to $374 \mathrm{~m}^{2} \mathrm{~g}^{-1}$ ). $S_{\mathrm{BET}}$ of the composite is still significantly below the value of pristine HKUST-1. However, the expected $S_{\mathrm{BET}}$ value, calculated from the contribution and surface areas of both parent phases, would be $360 \mathrm{~m}^{2} \mathrm{~g}^{-1}$. This is in good agreement with the measured $S_{\mathrm{BET}}$ and implies that HKUST-1 confined within the silica cavities is fully accessible for hosting $\mathrm{N}_{2}$ molecules. Additionally, the adsorption increase is more flattened with a less pronounced hysteresis loop with respect to the isotherm curve of the silica matrix indicating partial plugging of the silica mesopores. Micropores of the composite seem to be uniform, completely matching the average size of the bulk HKUST-1 but with narrower pore size distribution. On the other hand, the average size of the mesopores and their contribution within the composite are significantly reduced due to the filling with the MOF phase.

The above described results already strongly imply that HKUST-1 is crystallized in the confined manner within FDU-12 mesopores in the composite, rather than as a separate phase. In order to finally confirm these indications, additional analysis was performed by HR-TEM. Micrographs of the $\mathrm{NH}_{2}$-FDU-12 silica matrix show a highly ordered cubic arrangement of spherical mesopores with the estimated size of $20 \mathrm{~nm}$ which is in accordance with PSD analysis (Fig. 3a and b). On the other hand, micrographs of HKUST-1/FDU-12 show clearly visible dark spots which seem to be exclusively located within the mesopore cavities, while the cubic mesopore arrangement is preserved (Fig. 3c and d). The pronounced difference in image contrast between the matrix and the nanoparticles with the size of approximately $10 \mathrm{~nm}$ located within the pores indicates the difference in the elemental composition of these two phases. These nanoparticles correspond to the $\mathrm{CuO}$ (tenorite) phase as confirmed by selective area electron diffraction (Fig. S3†). The presence of $\mathrm{CuO}$ can be explained by the rapid degradation of HKUST-1 under a highly focused $200 \mathrm{kV}$ beam. Indeed, as it is the case for the majority of MOFs, HKUST-1 is beam sensitive and degradation can be observed almost instantly when it is exposed to high-energy electron beam. Even though the HKUST1 phase cannot be directly observed, the detection of homogeneously dispersed $\mathrm{CuO}$, located exclusively within the mesopores, by HRTEM analysis is an indirect proof that HKUST-1 is confined within the mesopores in the undamaged sample as well.

In order to gain more in-depth information about the structural correlation and interactions between the MOF phase and silica wall, additional studies were performed using NMR and EPR spectroscopic methods. Firstly, we employed ${ }^{29} \mathrm{Si}$ NMR measurements to observe the changes of the FDU-12 matrix during consecutive steps of the material preparation. ${ }^{29} \mathrm{Si}$ CPMAS spectra are presented in Fig. 4 and exhibit few significant differences. The ${ }^{29} \mathrm{Si}$ CPMAS spectrum of as-synthesized FDU-12 (Fig. 4, orange line) features three distinct signals at approximately $-90,-100$ and $-110 \mathrm{ppm}$ which can be assigned to Q2 $\left(\mathrm{Si}(\mathrm{OSi})_{2}(\mathrm{OH})_{2}\right)$, Q3 $\left(\mathrm{Si}(\mathrm{OSi})_{3}(\mathrm{OH})\right)$ and $\mathrm{Q} 4\left(\mathrm{Si}(\mathrm{OSi})_{4}\right)$ silica 
Table 1 Textural properties of the investigated samples obtained from $\mathrm{N}_{2}$ sorption isotherms

\begin{tabular}{lcllc}
\hline Sample & $S_{\mathrm{BET}}{ }^{a}\left(\mathrm{~m}^{2} \mathrm{~g}^{-1}\right)$ & $V_{\mathrm{mes}^{b}}\left(\mathrm{~cm}^{3} \mathrm{~g}^{-1}\right)$ & $V_{\mathrm{mic}^{c}}\left(\mathrm{~cm}^{3} \mathrm{~g}^{-1}\right)$ & $d_{\mathrm{mes}}^{d}(\mathrm{~nm})$ \\
\hline NH $_{2}$-FDU-12 & 72 & 0.68 & 0.05 & $8.8^{e} / 21.5^{f}$ \\
HKUST-1 & 1511 & - & 0.49 & $-d_{\mathrm{mic}}^{d}(\mathrm{~nm})$ \\
HKUST-1/FDU-12 & 374 & 0.54 & 0.38 & $4.1^{e} / 12.2^{f}$
\end{tabular}

${ }^{a}$ Specific surface area calculated by the BET method. ${ }^{b}$ Mesopore volume based on the BJH method. ${ }^{c}$ Micropore volume obtained from $\alpha$-plot calculations. ${ }^{d}$ Average pore size obtained from NLDFT PSD analyses. ${ }^{e}$ Pore openings. ${ }^{f}$ Cavities.

environments (Fig. 4, grey area), respectively. Rest of the spectra (spectra corresponding to APTES-functionalized materials) exhibit additional resonances at approximately -58 and $-67 \mathrm{ppm}$; such resonances are assigned to T2 $\left(\mathrm{RSi}(\mathrm{OSi})_{2}(\mathrm{OH})\right)$ and T3 $\left(\mathrm{RSi}(\mathrm{OSi})_{3}\right)$ silica environments (Fig. 4, yellow area). Noticeably, the relative density of the Q4 groups is similar (approximately 25\%) in all the spectra because such groups are not part of the functionalization process, i.e., they have no free silanols that can be replaced by APTES. In contrast, Q2 and Q3 groups comprise free silanols and can act as functionalization sites. Upon functionalization, the relative densities of Q2 and Q3 groups decrease as some of them have free silanols replaced by APTES molecules and therefore appear as T2 and T3 resonances. The decrease of the density of the $\mathrm{Q}$ environments and consequent increase of the density of the $\mathrm{T}$ environments clearly proves that the functionalization process was successful and APTES molecules were indeed bound to the silica walls of FDU-12.

Additionally, ${ }^{13} \mathrm{C}$ NMR measurements provided the information about HKUST-1 features. The detailed NMR study of bulk HKUST-1 was already performed by Dawson and coworkers. ${ }^{52}$ They have managed to unambiguously assign ${ }^{13} \mathrm{C}$ NMR resonances and their work served as a reference for our measurements. Results are shown in Fig. 5 where ${ }^{13} \mathrm{C}$ CPMAS spectra of $\mathrm{NH}_{2}$-FDU-12 and $\mathrm{Cu}-\mathrm{NH}_{2}$-FDU-12 are plotted alongside the ${ }^{13} \mathrm{C}$ spin-echo spectrum of HKUST-1/FDU-12. In the case of HKUST-1/FDU-12, the performance of the usually used CPMAS sequence suffered immensely due to the presence of paramagnetic $\mathrm{Cu}$ centers and was thus replaced by the spinecho sequence which gave better results. ${ }^{13} \mathrm{C}$ spectra of $\mathrm{NH}_{2}$ FDU-12 and Cu-NH${ }_{2}$-FDU-12 (Fig. 5, black and green lines, respectively) exhibit two distinct groups of signals; the first ones belong to the aliphatic carbons of APTES (yellow area) and the second ones to the probe background (grey area). More diverse is the spectrum of HKUST-1/FDU-12 (Fig. 5, red line) which comprises three groups of resonances. Again, aliphatic resonances of APTES (yellow area) are clearly visible alongside two distinct signals resonating at approx. -50 and $230 \mathrm{ppm}$ (green area) and a group of three signals ranging from 150 to $190 \mathrm{ppm}$ (blue area). The two green-highlighted signals with unusual isotropic shifts perfectly coincide with characteristic HKUST-1 signals reported in Dawson's study and are thus assigned to crystalline HKUST-1. Considering that the formation of HKUST1 occurred only within FDU-12 mesopores, we assigned these two signals to such nanoconfined crystalline HKUST-1. The reason that these two signals resonate at higher (230 ppm) or lower frequencies $(-50 \mathrm{ppm})$, as is usually expected for ${ }^{13} \mathrm{C}$ signals, is the strong paramagnetic influence of the $\mathrm{Cu}$ centers. Interestingly, Dawson and coworkers observed a HKUST-1 signal at even higher frequencies, namely, a broad and weak signal resonating at approximately $850 \mathrm{ppm}$. However, we did not observe such a signal in the HKUST-1/FDU-12 composite probably due to the much lower "density" of FDU-12-confined HKUST-1 compared to the bulky one, which renders the already weak signal practically undetectable. The most interesting aspect of the spectrum of HKUST-1/FDU-12 is the three blue-highlighted signals. The unpredictability of isotropic chemical shifts, due to the paramagnetic nature of the $\mathrm{Cu}$ nucleus, makes signal assignment almost impossible without some sort of precise sample manipulation (e.g. selective ${ }^{13} \mathrm{C}$ enrichment as in Dawson's study). Consequently, ${ }^{13} \mathrm{C}$ chemical shift values should be compared with those of a reliable precedent (as is the case with green-highlighted signals). Nevertheless, it is possible that these signals correspond to BTC ligands in a somewhat distorted configuration, e.g., ligands at the surface of HKUST-1 nanocrystals or ligands which are part of non-perfect paddle-wheel units. The paramagnetic effects felt by such ligands would certainly be different than the one felt by ligands inside crystalline HKUST and consequently their ${ }^{13} \mathrm{C}$ isotropical chemical shifts could change significantly. In order to gain additional information regarding the nature of the environment of copper nuclei, electron paramagnetic resonance analysis was performed.

The EPR spectra obtained at $77 \mathrm{~K}$ and $300 \mathrm{~K}$ for the bulk HKUST-1 and HKUST-1/FDU-12 are compared in Fig. 6, after normalization for the peak-to-peak amplitude of the main resonance lines. The EPR spectrum of bulk HKUST-1 acquired at $300 \mathrm{~K}$ shows a very broad and symmetric resonance. It has a peak-to-peak width of about $100 \mathrm{mT}$. A resonance with such spectroscopic properties is well known in the literature for bulk HKUST-1 and it is attributed to a paramagnetic center in the $S=$ 1 state, named $E^{\prime \prime}(\mathrm{Cu})$ center or simply triplet center. ${ }^{53,54}$ This center originates within the paddle-wheel metallic group, which consists of a couple of $\mathrm{Cu}^{2+}$ ions (each lying in the $S=1 / 2$ state) linked together by four carboxylate bridges. ${ }^{54,55}$ It was proved that the two $S=1 / 2$ of the two $\mathrm{Cu}^{2+}$ ions involved in the paddlewheels of HKUST-1 are coupled by antiferromagnetic interaction, generating a ground diamagnetic state with total spin $S$ $=0$ and an excited paramagnetic state with total spin $S=1$ and energy about $340 \mathrm{~cm}^{-1}$ above the ground state. ${ }^{55}$ The triplet center observed in Fig. 6a for bulk HKUST-1 is also supported by the EPR spectrum obtained for the same sample at $77 \mathrm{~K}$. 

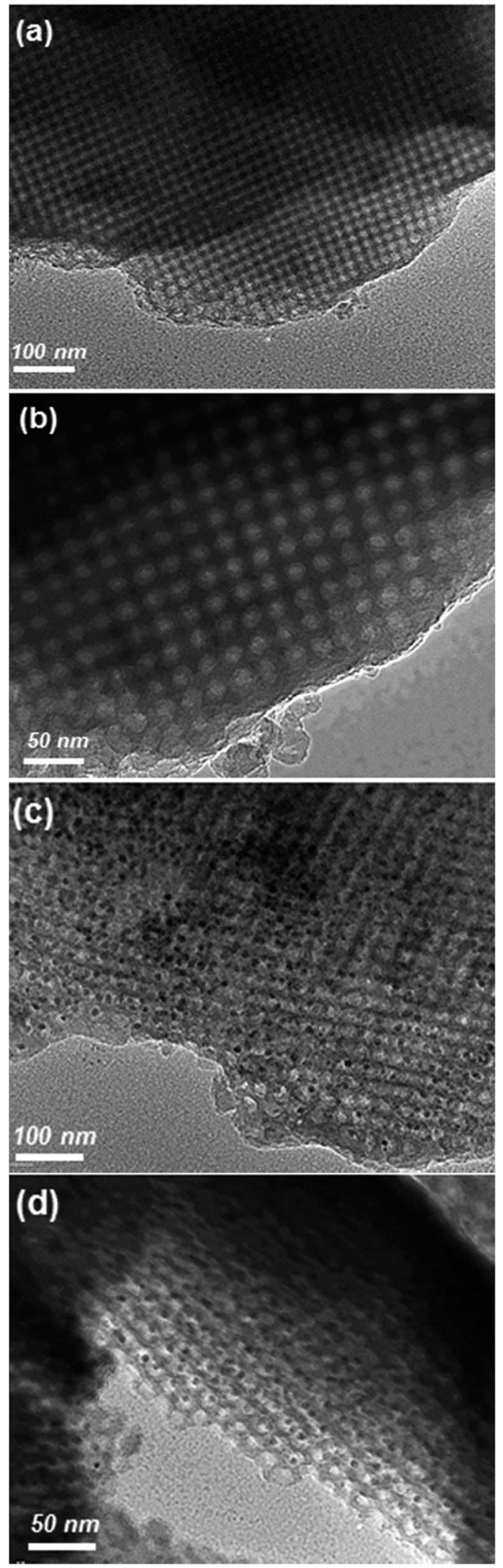

Fig. 3 HRTEM micrographs of (a, b) $\mathrm{NH}_{2}-\mathrm{FDU}-12$ silica matrix and (c, d) HKUST-1/FDU-12 composite viewed at lower $(a, c)$ and higher $(b, d)$ magnifications.

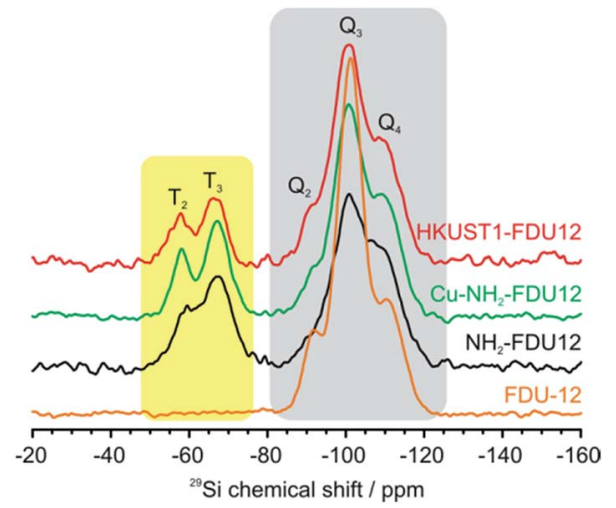

Fig. $4{ }^{1} \mathrm{H}_{-}{ }^{29} \mathrm{Si} \mathrm{CPMAS}$ NMR spectra of FDU-12 (orange), $\mathrm{NH}_{2}-\mathrm{FDU}-12$ (black), $\mathrm{Cu}-\mathrm{NH}_{2}-\mathrm{FDU}-12$ (green) and HKUST-1/FDU-12 (red). T and Q labels denote resonances corresponding to different $T$ (yellow area) and $Q$ (grey area) silica environments.

Characteristic peaks attributed to the $E^{\prime \prime}(\mathrm{Cu})$ center are well recognizable for magnetic fields of about 25 and $480 \mathrm{mT}$ (see arrows in Fig. 6b). ${ }^{56-58}$ The EPR signal of the triplet center obtained at $77 \mathrm{~K}$ has a significantly smaller amplitude than the one obtained at $300 \mathrm{~K}$. This feature is expected since the population of the excited $S=1$ magnetic state decreases exponentially with the decrease of temperature. The main central resonance observed in the spectrum of the bulk HKUST-1 in Fig. $6 \mathrm{~b}$ is attributable to $S=1 / 2$ paramagnetic centers, presumably due to the complexes involving monomeric $\mathrm{Cu}^{2+}$ ions or uncoupled $\mathrm{Cu}^{2+}$ pairs pertaining to somewhat distorted/ stressed paddle-wheels. ${ }^{53,55}$ Another interesting issue concerns the comparison of the resonance lineshapes observed for the

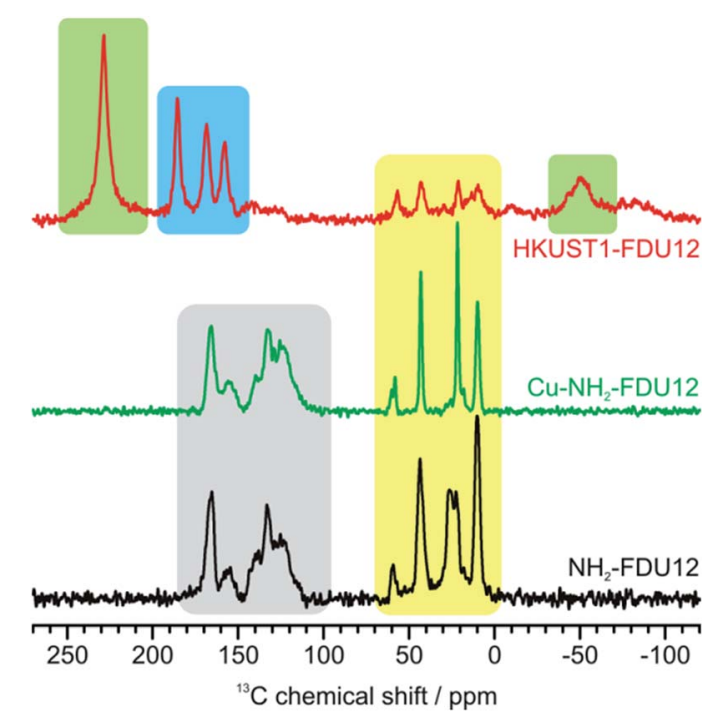

Fig. $5{ }^{13} \mathrm{C}$ NMR spectra of $\mathrm{NH}_{2}-\mathrm{FDU}-12$ (CPMAS, black), $\mathrm{Cu}-\mathrm{NH}_{2}-$ FDU-12 (CPMAS, green) and HKUST-1/FDU-12 (spin-echo, red). Coloured areas denote distinct groups of resonances: probe background (grey), APTES aliphatic carbons (yellow), two typical HKUST-1 resonances (green) and signals tentatively assigned to either BTC linkers at the surface of HKUST-1 nanocrystals or linkers in distorted Cu paddlewheel dimers (blue). 

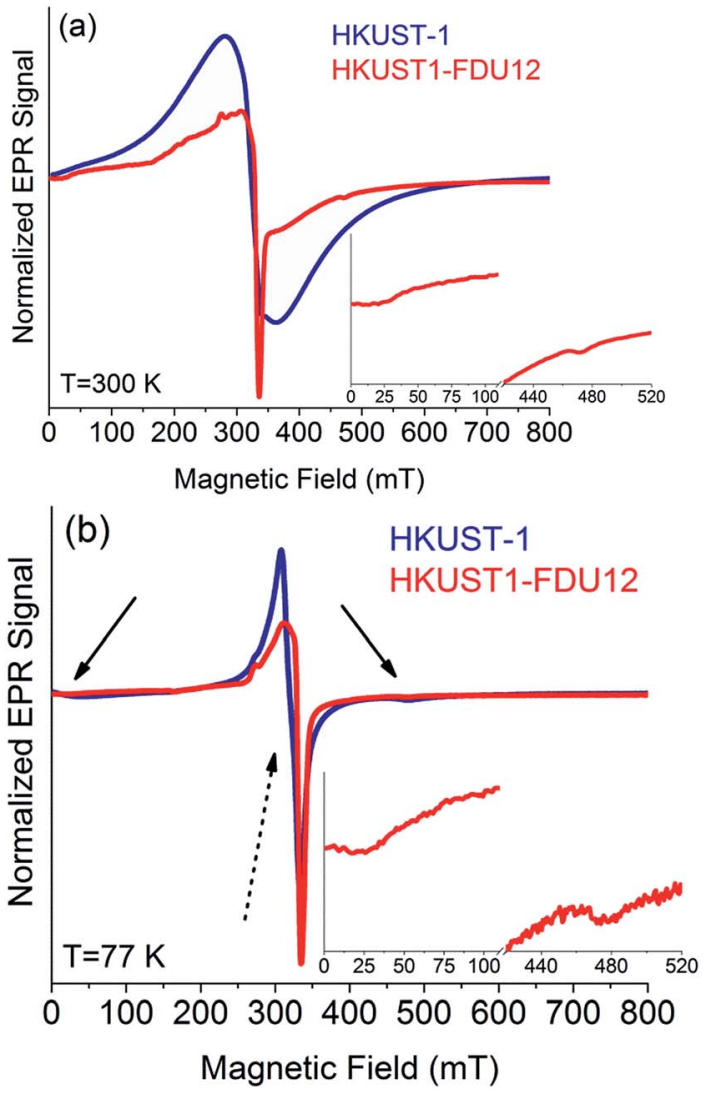

Fig. 6 Normalized EPR spectra of HKUST-1 bulk (blue line) and of HKUST-1/FDU-12 (red line) acquired at (a) $T=300 \mathrm{~K}$ and (b) $T=77 \mathrm{~K}$. The insets show zoomed images of the tails of the main resonance, providing evidence for the two small peaks present in the EPR spectrum of HKUST-1/FDU-12 for magnetic fields of about 25 and $480 \mathrm{mT}$. In (b) the spectral features attributable to the triplet centre are indicated by arrows, whereas those due to monomeric $\mathrm{Cu}^{2+}$ complexes and/or to uncoupled $\mathrm{Cu}^{2+}$ pairs by a dashed arrow.

triplet center at $77 \mathrm{~K}$ and $300 \mathrm{~K}$. As shown in Fig. 6 for bulk HKUST-1, the spectrum obtained at $300 \mathrm{~K}$ consists of a broad line at about $320 \mathrm{mT}$, whereas that obtained at $77 \mathrm{~K}$ involves two narrow resonances at about 25 and $480 \mathrm{mT}$. This difference is caused by the interaction among the neighbouring triplet centers, which makes the characteristic resonance of the triplet, observed at $77 \mathrm{~K}$, to collapse into a single broad line, as observed at $300 \mathrm{~K} .{ }^{59,60}$ This effect is induced at $300 \mathrm{~K}$ because the triplet state is much more populated than at $77 \mathrm{~K}$ and consequently the paddle-wheels giving rise to $S=1$ states are statistically closest to each other, significantly increasing the strength of their mutual interaction.

The EPR spectrum of HKUST-1/FDU-12 obtained at $77 \mathrm{~K}$ is comparable with that obtained for HKUST-1 bulk, as shown in Fig. 6b. In fact, for both materials we observe a signal arising from the triplet center (arrows) and a main resonance line due to $S=1 / 2$ species (dashed arrow), although the latter component is somewhat broader for HKUST-1/FDU-12 than for the bulk HKUST-1. This broadening effect indicates a more prominent inhomogeneous distribution of the paramagnetic centers in the former system than in the latter and it is presumably related to the nanometric confinement of HKUST-1 into the cavities of FDU-12 mesoporous silica. The EPR spectrum of HKUST-1/FDU-12, reported in Fig. 6a, presents a more structured lineshape. By the detailed analysis of the properties of this resonance we have recognized that it actually arises from the superposition of three distinguishable contributions, whose EPR lineshapes are shown in Fig. 7. A comparison of the experimental spectrum with the result of the linear combination, with appropriate weights, of these three component lines is presented in Fig. 7a. As shown, a very good agreement is found, strongly supporting our approach. In particular, component 1 is the lineshape corresponding to the $E^{\prime \prime}(\mathrm{Cu})$ center measured at $300 \mathrm{~K}$. It is the same lineshape we have observed for HKUST-1 bulk at the same temperature and indicates that HKUST-1/FDU-12 contains HKUST-1 with the usual magnetic properties. Component 2 is again attributable to the $E^{\prime \prime}(\mathrm{Cu})$ center, but it has the lineshape usually observed at $77 \mathrm{~K}$ for bulk HKUST-1. Its observation at $300 \mathrm{~K}$ for the HKUST-1/ FDU-12 sample is a very interesting and original result. In fact, it indicates that in the latter material a measurable fraction of the $S=1$ centers are not able to establish the characteristic interaction among neighbouring triplet centers which is responsible for the collapse of the lineshape into a single broad resonance, as discussed before. This contribution to the EPR spectrum is presumably due to the paddle-wheels located near the border of the nanometric HKUST- 1 crystals synthesized in the cavities of the mesoporous silica. In fact, due to their specific location, they naturally have a significantly reduced number of similar neighbouring paramagnetic centers. Finally, it is easy to recognize that Component 3 is attributable to the $S$ $=1 / 2 \mathrm{Cu}^{2+}$ species. Also, this contribution is presumably related to the nanometric confinement of HKUST-1 in the composite material. In fact, in such a material we expect that at the interface between the nanometric HKUST-1 crystal and the

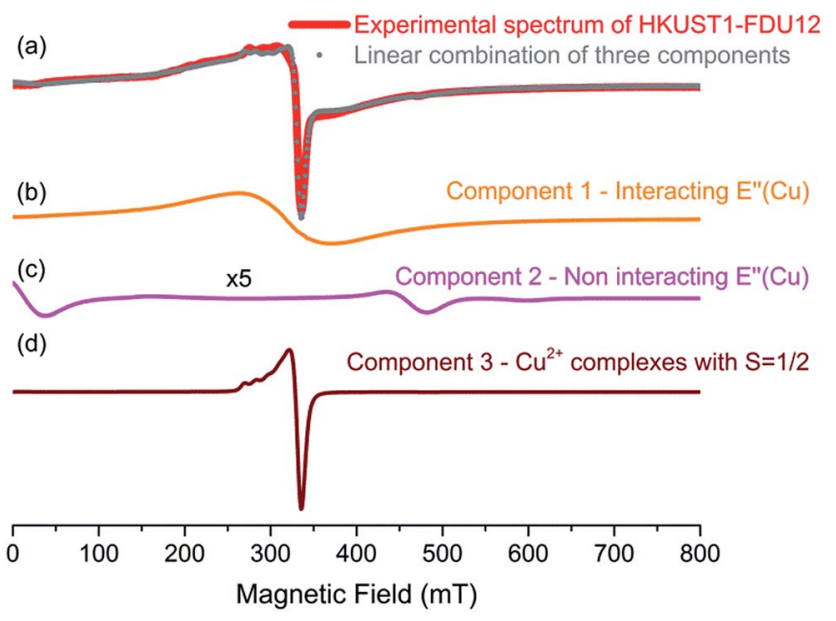

Fig. 7 (a) Comparison of the experimental spectrum of HKUST-1/ FDU-12 (red) with the result of the linear combination (grey circles) of the three components. (b) Component 1 (orange), arising from interacting $E^{\prime \prime}(\mathrm{Cu})$ centers. (c) Component 2 (magenta), magnified for better viewing $(5 \times)$, related to non-interacting $E^{\prime \prime}(\mathrm{Cu})$ centers. (d) Component 3 (wine), associated with $S=1 / 2 \mathrm{Cu}^{2+}$ species. 
internal surfaces of the silica a relevant number of incomplete and/or stressed paddle-wheels are present. Such structures are expected to manifest just as simple $S=1 / 2$ species, instead of $S$ $=1$ centers, in line with our observations.

Summarizing, the detailed analysis of the EPR spectra of HKUST-1/FDU-12 and its comparison with those recorded for HKUST-1 bulk indicates that the material synthesized inside the pores of FDU-12 is actually crystalline HKUST- 1 with essentially the usual structural and magnetic properties. In particular, the confinement of HKUST-1 crystals in nanosized pores is further supported by the original observation of a component in the EPR spectrum at $T=300 \mathrm{~K}$ attributable to paddle-wheels located near the border of the nanometric spatially confined HKUST-1 crystals.

\section{Sorption properties}

As shown by various characterization methods described above, HKUST-1 is successfully crystallized within the mesopore cavities of the $\mathrm{NH}_{2}$-FDU-12 matrix. However, it is reasonable to believe that the confinement will have negative effects on the accessibility of MOF sorption sites. The silica matrix could seriously hinder the sorption capacities of MOFs or even completely prevent the host molecules from entering into the micropores. Therefore, sorption capacities for different gases $\left(\mathrm{H}_{2}, \mathrm{CH}_{4}\right.$ and $\left.\mathrm{CO}_{2}\right)$ of HKUST-1/FDU-12 were compared with those of bulk HKUST-1 in order to evaluate the effect of silica 'coating' on the accessibility for gas molecules. The gas sorption capacities of HKUST-1 confined within the silica matrix remain almost unchanged. The uptakes are intentionally given in $\mathrm{cm}^{3}$ of adsorbed gas per $\mathrm{cm}^{3}$ of adsorbent in order to easily correlate the sorption capacities with the contribution of MOF in the composites. The volume uptakes for $\mathrm{CO}_{2}, \mathrm{CH}_{4}$ and $\mathrm{H}_{2}$ at high pressures decrease by approximately $50 \%$ (when complete saturation is more or less achieved) in all cases though, if compared with the bulk HKUST-1 which exhibits the expected uptakes (Fig. S4 $\dagger$ ). ${ }^{61-63}$ As already estimated by TG and $\mathrm{N}_{2}$ sorption analysis, one half of the FDU-12 mesopore cavities are filled with HKUST-1, implying that the decrease of sorption capacities in the case of the composite occurs mainly due to the presence of the FDU-12 silica matrix, whereas HKUST-1 confined within the mesopores seems to be completely accessible for gas molecules.

\section{Hydrostability}

In order to test the hydrostability of the MOF structure within the composite, the material was exposed to water under different conditions. As shown in Fig. 8, the bulk HKUST-1 structure is sensitive to moisture. Its crystallinity significantly decreases even after exposure to a controlled humid environment with $75 \%$ of relative humidity, and undergoes complete structural transformation into the nonporous $\left[\mathrm{Cu}_{2} \mathrm{OH}(\mathrm{BTC})\left(\mathrm{H}_{2} \mathrm{O}\right)\right] \cdot 2 \mathrm{H}_{2} \mathrm{O}$ phase ${ }^{64}$ after stirring in water. On the other hand, the composite structure remains intact even after direct contact with water at $50{ }^{\circ} \mathrm{C}$ for 1 day. Moreover, in contrast to bulk MOF, the exposure to water does not notably affect the porosity in the case of the composite, as indicated by
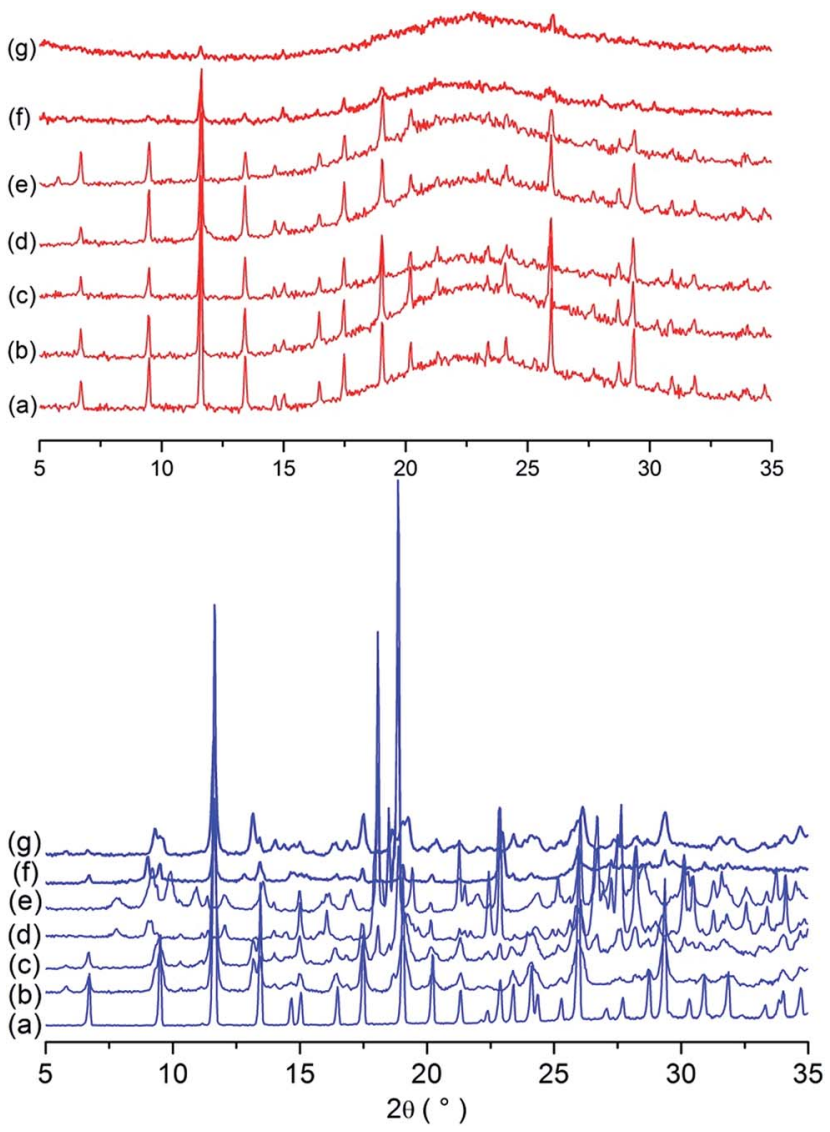

Fig. 8 XRD patterns of the HKUST-1/FDU-12 (above) and bulk HKUST1 (below) after water exposure under different conditions compared with the (a) as-synthesized materials: (b) 3 days at $75 \% \mathrm{RH}$; (c) 3 days at $98 \% \mathrm{RH}$; (d) stirring in water at room temperature overnight; (e) stirring in water at $50{ }^{\circ} \mathrm{C}$ overnight; (f) stirring in $0.05 \mathrm{M} \mathrm{NaOH}$; (g) stirring in $0.05 \mathrm{M} \mathrm{HCl}$.

the $\mathrm{N}_{2}$ sorption isotherm measured after stirring in water at room temperature overnight (Fig. S5 $\dagger$ ). The BET specific surface area of the composite decreases by about 7\% (from $569 \mathrm{~m}^{2} \mathrm{~g}^{-1}$ to $529 \mathrm{~m}^{2} \mathrm{~g}^{-1}$ ), whereas in the case of the bulk HKUST-1, the surface area drop is significantly larger (from $1211 \mathrm{~m}^{2} \mathrm{~g}^{-1}$ to 215 $\mathrm{m}^{2} \mathrm{~g}^{-1}$ ). The reason for the enhanced structural resistivity to water may lie in the hydrophobic nature of the silica FDU-12 matrix, in which HKUST-1 is crystallized in the confined manner. As reported previously using ${ }^{29} \mathrm{Si}$ MAS NMR investigations, the high temperature of the FDU-12 synthesis (180-200 $\left.{ }^{\circ} \mathrm{C}\right)$ significantly enhances the condensation of terminal silanol groups (Q3 and Q2) into Q4, and thus increases the hydrophobic nature of the silica framework. ${ }^{65}$ This provides a protective environment for HKUST-1 and, at least to some degree, repels the water molecules from the MOF structure. The lower hydrophilicity of the composite in comparison with the bulk HKUST-1 is implied by water sorption isotherm measurements at $25{ }^{\circ} \mathrm{C}$. The isotherms indeed indicate that the composite does not have any notable affinity to adsorb water in contrast to pure HKUST-1 which exhibits the expected high water uptake ${ }^{66}$ (Fig. S6 $†$ ). The water-repelling role of the FDU-12 matrix can also be seen from the isotherm with the water uptake 
normalized to the HKUST-1 content within the composite. The normalized water sorption capacity up to relative pressures of 0.8 reaches only approximately $30 \%$ of the values of pristine HKUST-1. More pronounced uptake is achieved only at higher pressures and can be attributed to the physisorption on the surface of the composite. As expected, the MOF structure in the composite becomes significantly damaged only under harsher, non-neutral conditions $(0.05 \mathrm{M} \mathrm{NaOH}-\mathrm{pH}=12 ; 0.05 \mathrm{M} \mathrm{HCl}-$ $\mathrm{pH}=1.5)$ as indicated by the XRD patterns in Fig. 8. There are two separate reasons for the decreased structural stabilities under such conditions. The higher solubility of HKUST-1 at lower $\mathrm{pH}$ causes pronounced leaching of the MOF phase out of the silica matrix. The instability of the silica FDU-12 matrix at higher $\mathrm{pH}$ values, on the other hand, causes partial disordering of the silica cubic mesostructure and consequently the collapse of the composite structure.

\section{Conclusions}

With the integration of a water-sensitive copper-based metalorganic framework (HKUST-1) in the mesopores of the FDU-12 silica matrix we aimed to design a MOF composite with enhanced hydrostability of the Cu-BTC framework. Using a twostep crystallization which includes $\mathrm{Cu}^{2+}$ impregnation in the first step, followed by MOF formation in the second step, HKUST1 loading of $20 \mathrm{wt} \%$ was achieved. With the in-depth structural studies we proved that the HKUST-1 framework is exclusively formed within the spherical cavities of the silica matrix. Structural correlation between the two phases was additionally evaluated by NMR and EPR spectroscopies. Spectra of the composite obtained by both methods exhibit unique features which are not found in the bulk MOF and could be assigned to the interrupted paddle-wheel species located on the crystallite boundaries.

In spite of the HKUST-1 confinement within the silica matrix, its micropores remain highly accessible for gas molecules. The decrease of the $\mathrm{H}_{2}, \mathrm{CO}_{2}$ and $\mathrm{CH}_{4}$ gas sorption capacities by approximately $50 \%$ with respect to the bulk MOF could be attributed mainly to the presence of the silica matrix. Moreover, HKUST-1 integrated within the silica matrix mesopores exhibits significantly improved structural stability upon water exposure in comparison with the bulk material. The MOF structure in the composite withstands the immersion and stirring overnight in water even at elevated temperature in contrast with the bulk HKUST-1 being highly prone to hydrolysis on direct contact with water. Confinement in the hydrophobic environment of the silica matrix to some extent prevents the diffusion of water within the mesostructure and protects the MOF from degradation.

The concept of integration into FDU-12 or related silica matrices, that are able to provide high accessibility of sorption sites and at the same time repel water from the frameworks, can also be expanded to other water-sensitive MOFs and enable them to selectively and sustainably capture humid gases.

\section{Conflicts of interest}

There are no conflicts of interest to declare.

\section{Acknowledgements}

This work was supported by the Slovenian Research Agency (grant P1-0021). The authors thank the people of the LAMP group (www.unipa.it/lamp) at the Department of Physics and Chemistry of the University of Palermo for stimulating discussions.

\section{Notes and references}

1 J. Canivet, A. Fateeva, Y. Guo, B. Coasne and D. Farrusseng, Water Adsorption in MOFs: Fundamentals and Applications, Chem. Soc. Rev., 2014, 43(16), 5594-5617.

2 J. Gascon, A. Corma, F. Kapteijn and F. X. Llabrés i Xamena, Metal Organic Framework Catalysis: Quo Vadis ?, ACS Catal., 2014, 4(2), 361-378.

3 N. A. Khan, Z. Hasan and S. H. Jhung, Adsorptive Removal of Hazardous Materials Using Metal-Organic Frameworks (MOFs): A Review, J. Hazard. Mater., 2013, 244-245, 444-456.

4 J.-R. Li, J. Sculley and H.-C. Zhou, Metal-Organic Frameworks for Separations, Chem. Rev., 2012, 112(2), 869932.

5 J. Liu, L. Chen, H. Cui, J. Zhang, L. Zhang and C.-Y. Su, Applications of Metal-Organic Frameworks in Heterogeneous Supramolecular Catalysis, Chem. Soc. Rev., 2014, 43(16), 6011-6061.

6 S. T. Meek, J. A. Greathouse and M. D. Allendorf, MetalOrganic Frameworks: A Rapidly Growing Class of Versatile Nanoporous Materials, Adv. Mater., 2011, 23(2), 249-267.

7 M. P. Suh, H. J. Park, T. K. Prasad and D.-W. Lim, Hydrogen Storage in Metal-Organic Frameworks, Chem. Rev., 2012, 112(2), 782-835.

8 K. Leus, T. Bogaerts, J. De Decker, H. Depauw, K. Hendrickx, H. Vrielinck, V. Van Speybroeck and P. Van Der Voort, Systematic Study of the Chemical and Hydrothermal Stability of Selected "stable" Metal Organic Frameworks, Microporous Mesoporous Mater., 2016, 226, 110-116.

9 J. J. Low, A. I. Benin, P. Jakubczak, J. F. Abrahamian, S. A. Faheem and R. R. Willis, Virtual High Throughput Screening Confirmed Experimentally: Porous Coordination Polymer Hydration, J. Am. Chem. Soc., 2009, 131(43), 15834-15842.

10 I. J. Kang, N. A. Khan, E. Haque and S. H. Jhung, Chemical and Thermal Stability of Isotypic Metal-Organic Frameworks: Effect of Metal Ions, Chem.-Eur. J., 2011, 17(23), 6437-6442.

11 J. H. Cavka, S. Jakobsen, U. Olsbye, N. Guillou, C. Lamberti, S. Bordiga and K. P. Lillerud, A New Zirconium Inorganic Building Brick Forming Metal Organic Frameworks with Exceptional Stability, J. Am. Chem. Soc., 2008, 130(42), 13850-13851.

12 B. Van de Voorde, D. Damasceno Borges, F. Vermoortele, R. Wouters, B. Bozbiyik, J. Denayer, F. Taulelle, C. Martineau, C. Serre, G. Maurin and D. De Vos, Isolation of Renewable Phenolics by Adsorption on Ultrastable Hydrophobic MIL-140 Metal-Organic Frameworks, ChemSusChem, 2015, 8(18), 3159-3166. 
13 H. Jasuja, N. C. Burtch, Y. Huang, Y. Cai and K. S. Walton, Kinetic Water Stability of an Isostructural Family of ZincBased Pillared Metal-Organic Frameworks, Langmuir, 2013, 29(2), 633-642.

14 S. H. Jhung, N. A. Khan and Z. Hasan, Analogous Porous Metal-Organic Frameworks: Synthesis, Stability and Application in Adsorption, CrystEngComm, 2012, 14(21), 7099.

15 P. M. Schoenecker, C. G. Carson, H. Jasuja, C. J. J. Flemming and K. S. Walton, Effect of Water Adsorption on Retention of Structure and Surface Area of Metal-Organic Frameworks, Ind. Eng. Chem. Res., 2012, 51(18), 6513-6519.

16 V. Colombo, S. Galli, H. J. Choi, G. D. Han, A. Maspero, G. Palmisano, N. Masciocchi and J. R. Long, High Thermal and Chemical Stability in Pyrazolate-Bridged MetalOrganic Frameworks with Exposed Metal Sites, Chem. Sci., 2011, 2(7), 1311.

17 H. J. Choi, M. Dincă, A. Dailly and J. R. Long, Hydrogen storage in Water-Stable Metal-Organic Frameworks Incorporating 1,3- and 1,4-Benzenedipyrazolate, Energy Environ. Sci., 2010, 3(1), 117-123.

18 N. C. Burtch, H. Jasuja and K. S. Walton, Water Stability and Adsorption in Metal-Organic Frameworks, Chem. Rev., 2014, 114(20), 10575-10612.

19 H. Jasuja, Y. Huang and K. S. Walton, Adjusting the Stability of Metal-Organic Frameworks under Humid Conditions by Ligand Functionalization, Langmuir, 2012, 28(49), 1687416880.

20 J. M. Taylor, R. Vaidhyanathan, S. S. Iremonger and G. K. H. Shimizu, Enhancing Water Stability of MetalOrganic Frameworks via Phosphonate Monoester Linkers, J. Am. Chem. Soc., 2012, 134(35), 14338-14340.

21 D. Ma, Y. Li and Z. Li, Tuning the Moisture Stability of Metal-Organic Frameworks by Incorporating Hydrophobic Functional Groups at Different Positions of Ligands, Chem. Commun., 2011, 47(26), 7377.

22 T. Wittmann, R. Siegel, N. Reimer, W. Milius, N. Stock and J. Senker, Enhancing the Water Stability of Al-MIL-101- $\mathrm{NH}_{2}$ via Postsynthetic Modification, Chem.-Eur. J., 2015, 21(1), 314-323.

23 T. Wu, L. Shen, M. Luebbers, C. Hu, Q. Chen, Z. Ni and R. I. Masel, Enhancing the Stability of Metal-Organic Frameworks in Humid Air by Incorporating Water Repellent Functional Groups, Chem. Commun., 2010, 46(33), 6120.

24 J. G. Nguyen and S. M. Cohen, Moisture-Resistant and Superhydrophobic Metal-Organic Frameworks Obtained via Postsynthetic Modification, J. Am. Chem. Soc., 2010, 132(13), 4560-4561.

25 J. Yang, A. Grzech, F. M. Mulder and T. J. Dingemans, Methyl Modified MOF-5: A Water Stable Hydrogen Storage Material, Chem. Commun., 2011, 47(18), 5244.

26 T.-H. Chen, I. Popov, O. Zenasni, O. Daugulis and O. Š. Miljanić, Superhydrophobic Perfluorinated MetalOrganic Frameworks, Chem. Commun., 2013, 49(61), 6846.

27 C. Serre, Superhydrophobicity in Highly Fluorinated Porous Metal-Organic Frameworks, Angew. Chem., Int. Ed., 2012, 51(25), 6048-6050.
28 T. Li, D.-L. Chen, J. E. Sullivan, M. T. Kozlowski, J. K. Johnson and N. L. Rosi, Systematic Modulation and Enhancement of CO2: N2 Selectivity and Water Stability in an Isoreticular Series of Bio-MOF-11 Analogues, Chem. Sci., 2013, 4(4), 1746.

29 Y. Cai, Y. Zhang, Y. Huang, S. R. Marder and K. S. Walton, Impact of Alkyl-Functionalized BTC on Properties of Copper-Based Metal-Organic Frameworks, Cryst. Growth Des., 2012, 12(7), 3709-3713.

30 T. A. Makal, X. Wang and H.-C. Zhou, Tuning the Moisture and Thermal Stability of Metal-Organic Frameworks through Incorporation of Pendant Hydrophobic Groups, Cryst. Growth Des., 2013, 13(11), 4760-4768.

31 S. J. Yang and C. R. Park, Preparation of Highly MoistureResistant Black-Colored Metal Organic Frameworks, Adv. Mater., 2012, 24(29), 4010-4013.

32 C. A. Fernandez, S. K. Nune, H. V. Annapureddy, L. X. Dang, B. P. McGrail, F. Zheng, E. Polikarpov, D. L. King, C. Freeman and K. P. Brooks, Hydrophobic and MoistureStable Metal-Organic Frameworks, Dalton Trans., 2015, 44(30), 13490-13497.

33 Y. Yoo, V. Varela-Guerrero and H.-K. Jeong, Isoreticular Metal-Organic Frameworks and Their Membranes with Enhanced Crack Resistance and Moisture Stability by Surfactant-Assisted Drying, Langmuir, 2011, 27(6), 26522657.

34 H. Li, W. Shi, K. Zhao, H. Li, Y. Bing and P. Cheng, Enhanced Hydrostability in Ni-Doped MOF-5, Inorg. Chem., 2012, 51(17), 9200-9207.

35 S. Li and F. Huo, Metal-Organic Framework Composites: From Fundamentals to Applications, Nanoscale, 2015, 7(17), 7482-7501.

36 Q.-L. Zhu and Q. Xu, Metal-Organic Framework Composites, Chem. Soc. Rev., 2014, 43(16), 5468-5512.

37 A. Kondo, S. Takanashi and K. Maeda, New Insight into Mesoporous Silica for Nano Metal-Organic Framework, $J$. Colloid Interface Sci., 2012, 384(1), 110-115.

38 Z. Ulker, I. Erucar, S. Keskin and C. Erkey, Novel Nanostructured Composites of Silica Aerogels with a Metal Organic Framework, Microporous Mesoporous Mater., 2013, 170, 352-358.

39 A. Chakraborty and T. K. Maji, Mg-MOF-74@SBA-15 Hybrids: Synthesis, Characterization, and Adsorption Properties, APL Mater., 2014, 2(12), 124107.

40 C.-M. Wu, M. Rathi, S. P. Ahrenkiel, R. T. Koodali and Z. Wang, Facile Synthesis of MOF-5 Confined in SBA-15 Hybrid Material with Enhanced Hydrostability, Chem. Commun., 2013, 49(12), 1223.

41 N. E. Tari, A. Tadjarodi, J. Tamnanloo and S. Fatemi, Synthesis and Property Modification of MCM-41 Composited with $\mathrm{Cu}(\mathrm{BDC}) \mathrm{MOF}$ for Improvement of $\mathrm{CO} 2$ Adsorption Selectivity, J. $\mathrm{CO}_{2}$ Util., 2016, 14, 126-134.

42 Y.-K. Seo, J. W. Yoon, U.-H. Lee, Y. K. Hwang, C.-H. Jun and J.-S. Chang, Formation of a Nanohybrid Composite between Mesostructured Cellular Silica Foam and Microporous Copper Trimesate, Microporous Mesoporous Mater., 2012, 155, 75-81. 
43 O. Shekhah, L. Fu, R. Sougrat, Y. Belmabkhout, A. J. Cairns, E. P. Giannelis and M. Eddaoudi, Successful Implementation of the Stepwise Layer-by-Layer Growth of MOF Thin Films on Confined Surfaces: Mesoporous Silica Foam as a First Case Study, Chem. Commun., 2012, 48(93), 11434.

44 A. Sachse, R. Ameloot, B. Coq, F. Fajula, B. Coasne, D. De Vos and A. Galarneau, In Situ Synthesis of Cu-BTC (HKUST-1) in Macro-/Mesoporous Silica Monoliths for Continuous Flow Catalysis, Chem. Commun., 2012, 48(39), 4749.

45 C. Xin, X. Jiao, Y. Yin, H. Zhan, H. Li, L. Li, N. Zhao, F. Xiao and W. Wei, Enhanced $\mathrm{CO}_{2}$ Adsorption Capacity and Hydrothermal Stability of HKUST-1 via Introduction of Siliceous Mesocellular Foams (MCFs), Ind. Eng. Chem. Res., 2016, 55(29), 7950-7957.

46 H. H. Delavari, H. R. Madaah Hosseini and A. Simchi, Effects of Particle Size, Shape and Crystal Structure on the Formation Energy of Schottky Vacancies in Free-Standing Metal Nanoparticles: A Model Study, Phys. B, 2011, 406(20), 3777-3780.

47 N. T. K. Thanh, N. Maclean and S. Mahiddine, Mechanisms of Nucleation and Growth of Nanoparticles in Solution, Chem. Rev., 2014, 114(15), 7610-7630.

48 H. Zhang and J. F. Banfield, Energy Calculations Predict Nanoparticle Attachment Orientations and Asymmetric Crystal Formation, J. Phys. Chem. Lett., 2012, 3(19), 28822886.

49 L. Huang, X. Yan and M. Kruk, Synthesis of Ultralarge-Pore FDU-12 Silica with Face-Centered Cubic Structure, Langmuir, 2010, 26(18), 14871-14878.

50 N. A. Khan, E. Haque and S. H. Jhung, Rapid Syntheses of a Metal-Organic Framework Material $\mathrm{Cu}_{3}(\mathrm{BTC})_{2}\left(\mathrm{H}_{2} \mathrm{O}\right)_{3}$ under Microwave: A Quantitative Analysis of Accelerated Syntheses, Phys. Chem. Chem. Phys., 2010, 12(11), 2625.

51 J. Landers, G. Y. Gor and A. V. Neimark, Density Functional Theory Methods for Characterization of Porous Materials, Colloids Surf., A, 2013, 437, 3-32.

52 D. M. Dawson, L. E. Jamieson, M. I. H. Mohideen, A. C. McKinlay, I. A. Smellie, R. Cadou, N. S. Keddie, R. E. Morris and S. E. Ashbrook, High-Resolution SolidState ${ }^{13} \mathrm{C}$ NMR Spectroscopy of the Paramagnetic MetalOrganic Frameworks, STAM-1 and HKUST-1, Phys. Chem. Chem. Phys., 2013, 15(3), 919-929.

53 M. Todaro, G. Buscarino, L. Sciortino, A. Alessi, F. Messina, M. Taddei, M. Ranocchiari, M. Cannas and F. M. Gelardi, Decomposition Process of Carboxylate MOF HKUST-1 Unveiled at the Atomic Scale Level, J. Phys. Chem. C, 2016, 120(23), 12879-12889.

54 S. S. A. Chui, Chemically Functionalizable Nanoporous Material $\left[\mathrm{Cu}_{3}(\mathrm{TMA})_{2}\left(\mathrm{H}_{2} \mathrm{O}\right)_{3}\right]_{n}$, Science, 1999, 283(5405), 1148-1150.

55 A. Pöppl, S. Kunz, D. Himsl and M. Hartmann, CW and Pulsed ESR Spectroscopy of Cupric Ions in the Metal-
Organic Framework Compound $\mathrm{Cu}_{3}(\mathrm{BTC})_{2}, J$. Phys. Chem. C, 2008, 112(7), 2678-2684.

56 H. EL Mkami, M. I. H. Mohideen, C. Pal, A. McKinlay, O. Scheimann and R. E. Morris, EPR and Magnetic Studies of a Novel Copper Metal Organic Framework (STAM-I), Chem. Phys. Lett., 2012, 544, 17-21.

57 B. Bleaney and K. D. Bowers, Anomalous Paramagnetism of Copper Acetate, Proc. R. Soc. A, 1952, 214(1119), 451-465.

58 E. Wasserman, L. C. Snyder and W. A. Yager, ESR of the Triplet States of Randomly Oriented Molecules, J. Chem. Phys., 1964, 41(6), 1763.

59 M. Šimènas, M. Kobalz, M. Mendt, P. Eckold, H. Krautscheid, J. Banys and A. Pöppl, Synthesis, Structure, and Electron Paramagnetic Resonance Study of a Mixed Valent Metal-Organic Framework Containing $\mathrm{Cu}_{2}$ PaddleWheel Units, J. Phys. Chem. C, 2015, 119(9), 4898-4907.

60 L. M. B. Napolitano, O. R. Nascimento, S. Cabaleiro, J. Castro and R. Calvo, Isotropic and Anisotropic Spin-Spin Interactions and a Quantum Phase Transition in a Dinuclear $\mathrm{Cu}(\mathrm{II})$ Compound, Phys. Rev. B: Condens. Matter Mater. Phys., 2008, 77(21), 214423.

61 B. Xiao, P. S. Wheatley, X. Zhao, A. J. Fletcher, S. Fox, A. G. Rossi, I. L. Megson, S. Bordiga, L. Regli, K. M. Thomas and R. E. Morris, High-Capacity Hydrogen and Nitric Oxide Adsorption and Storage in a MetalOrganic Framework, J. Am. Chem. Soc., 2007, 129(5), 12031209.

62 H. Wu, J. M. Simmons, Y. Liu, C. M. Brown, X.-S. Wang, S. Ma, V. K. Peterson, P. D. Southon, C. J. Kepert, H.-C. Zhou, T. Yildirim and W. Zhou, Metal-Organic Frameworks with Exceptionally High Methane Uptake: Where and How Is Methane Stored?, Chem.-Eur. J., 2010, 16(17), 5205-5214.

63 J. Moellmer, A. Moeller, F. Dreisbach, R. Glaeser and R. Staudt, High Pressure Adsorption of Hydrogen, Nitrogen, Carbon Dioxide and Methane on the MetalOrganic Framework HKUST-1, Microporous Mesoporous Mater., 2011, 138(1-3), 140-148.

64 M. Schlesinger, S. Schulze, M. Hietschold and M. Mehring, Evaluation of Synthetic Methods for Microporous MetalOrganic Frameworks Exemplified by the Competitive Formation of $\left[\mathrm{Cu}_{2}(\mathrm{btc})_{3}\left(\mathrm{H}_{2} \mathrm{O}\right)_{3}\right]$ and $\left[\mathrm{Cu}_{2}(\mathrm{btc})(\mathrm{OH})\left(\mathrm{H}_{2} \mathrm{O}\right)\right]$, Microporous Mesoporous Mater., 2010, 132(1-2), 121-127.

65 G. Lawrence, A. V. Baskar, M. H. El-Newehy, W. S. Cha, S. S. Al-Deyab and A. Vinu, Quick High-Temperature Hydrothermal Synthesis of Mesoporous Materials with 3D Cubic Structure for the Adsorption of Lysozyme, Sci. Technol. Adv. Mater., 2015, 16(2), 024806.

66 P. Küsgens, M. Rose, I. Senkovska, H. Fröde, A. Henschel, S. Siegle and S. Kaskel, Characterization of Metal-Organic Frameworks by Water Adsorption, Microporous Mesoporous Mater., 2009, 120(3), 325-330. 